\title{
OPERATION OF THE RHIC RF SYSTEMS*
}

\author{
J.M. Brennan ${ }^{\#}$, M. Blaskiewicz, J. DeLong, W. Fischer, T. Hayes, K.S. Smith, A. Zaltsman, \\ Brookhaven National Laboratory \\ Upton, NY11973, USA
}

\section{Abstract}

Operational aspects of the RHIC if system are described. To date three different beam combinations have been collided for physics production: gold-gold, deuterongold, and proton-proton(polarized).[1,2] To facilitate this flexibility the rf systems of the two rings are independent and self-sufficient.[3] Techniques to cope with problems such as, injection/capture, beam loading, bunch shortening, and rf noise have evolved and are explained.

\section{INJECTION}

The injector (AGS) holds the beam on a magnetic tlattop (10 GeV/n for ions, $26 \mathrm{GeV}$ for protons) for about 1 second while synchronization and bunch-by-bunch extraction to RHIC take place. Since single bunches are extracted any bunch filling pattern is possible. Typically 6,60 , or 120 bunch patterns are used and a $1 \mu$ gap is left for the abort kicker risetime.

\section{Cogging in the AGS}

The circumference ratio between the RHIC rings and the AGS is 19:4. This means that 19 times the RHIC revolution frequency equals the AGS bunch frequency when it has 4 bunches. Also every 4 turns of RHIC the same bunch aligns with a given bucket in RHIC. The hardware[4] that orchestrates the fill process is composed of four Direct Digital Synthesizers which share a common source clock and are synchronously started on an "cogging reset" event which precedes each fill. Table 1 list the clocks and gives their function. The phases of the kicker trigger and synchro reference cloek advance appropriately between bunch transfers. Since the bunches are locked to the synchro reference clock they follow its phase change by making a radial (frequency) excursion. A Time Interval analyzer monitors and logs the time between the bunch and the kicker at each extraction.

\begin{tabular}{|l|l|l|}
\hline clock & $\begin{array}{l}\text { Frequency } \\
\text { (RHIC) }\end{array}$ & function \\
\hline RevTick & 1 Frevolution & Marks bunch one \\
\hline Kicker trig & $1 / 4$ Frev & Fires the kickers \\
\hline Synchro Ref & 19 Frev & Bunches lock to this \\
\hline $\begin{array}{l}\text { Beam Syne } \\
\text { clock. }\end{array}$ & 360 Frev & $\begin{array}{l}\text { Source for beam synch } \\
\text { clock systern }\end{array}$ \\
\hline
\end{tabular}

Table 1. Clocks of the injector synchronization system

\footnotetext{
Work performed under contract DE-AC02-98CH10886 with US DOE. "brennan@bol.gov
}

\section{Capture}

Successful rf capture in founded in a precise measurement of the beam revolution frequency with the rf off. We inject a single bunch and measure the revolution frequency using data from a deep-memory oscilloscope. When the beam was injected the synchronization circuit in the AGS was responding to the RHIC clocks (Table 1) which were set to the best guess for the capture frequency. After the measurement the frequency of the clocks is corrected. In order not to change the beam energy, and hence revolution frequency, the AGS flattop field is adjusted according to, $\left(\frac{\Delta B}{B}\right)_{A O S}=\left(\gamma_{t r}^{2}\right)_{A G S}\left(\frac{\Delta f}{f}\right)_{R H C}$, where $\Delta f$ is the correction. The bunches of the two rings are separated longitudinally at injection to prevent beam-beam interaction. This requires that the two rings have exactly the same capture frequency and that the phase of RevTick clocks are preset. The exact frequency mateh can be achieved with no more than $1 \mathrm{~mm}$ difference in average orbit radius between the rings. The phase of the 19 Frev synchro reference clock is adjusted to insure that the beam is injected into the center of the RHIC rf buckets.

\section{RAMPS}

The ramp rate to $100 \mathrm{GeV} /$ nucleon is modest $(\mathrm{d} \gamma / \mathrm{d} t<1)$ because the magnets are superconducting. A constant if voltage of about $300 \mathrm{kV}$ is supplied by two cavities which operate at a synchronous phase of less than 4 degrees. The voltage requirement follows from the necessary bucket area of $>1 \mathrm{eVs} / \mathrm{n}$. The ion beams must cross the transition energy and the machine is equipped with fast transition-jump quadrupoles. The only special demand for the rf system is to provide a smooth phase reference to the beam control loops during the phase jump of the cavities.

\section{Acceleration Cavities}

The acceleration cavities are equipped with direct rf feedback with $>38 \mathrm{~dB}$ gain. This feedback is essential for reducing the effective impedance below the threshold for instability. The practical gain is not limited by loop delay but by transients that occur when the carity is, switched on/off, phase jump at transition, or multipactoring. The driver amplifiers have been upgraded to units that tolerate mismatch during transients. Cavities need to be conditioned against multipactoring for several days before the run starts. Gradual de-conditioning occurs over a time scale of months but the rf feedback helps break through multipactoring. To condition the cavities the rf feedback loop is opened via a remotely controlled transfer switch. 


\section{Beam Control Feedback Loops}

Ramping is normally performed with conventional phase and radial beam control loops. [5] For crossing the gamma transition the radial loop is a must. The phase loop damps coherent dipole oscillations and minimizes emittance growth during the ramp. The radial loop determines the precise value of frequency, which changes by $0.4 \%$ for ions during the ramp.

The radial loop samples the beam position at two beam position monitors in an arc that are separated by approximately $1 / 3$ betatron wavelength. To a first approximation this technique separates the effect of orbit distortion from average orbit changes. Experience has shown, however, that this is often not sufficient and the average of these two BPMs differs from the average of the entire orbit. In practice a dipole corrector between the two BPMs is "tweaked" to compensate.

The heart to the beam control system is a DSP (Texas Instruments $\mathrm{C} 40$ ) that reads the error signals and makes corrections to the if frequency. The radius signal is sampled by a 16 bit $\mathrm{ADC}$, clocked at the revolution frequency $(78 \mathrm{kHz})$. The analog signal is generated in a homodyne detector with intensity normalization by implicit division in an $A G C$ loop. A low-noise $1 \mathrm{kHz}$ analog anti-aliasing filter precedes the ADC.

Phase data come from the bunch-by-bunch phase detector that measures every bunch on every turn. I and $Q$ signals are obtained with 14 bit ADCs and converted to magnitude and phase using the "CORDIC" algorithm[6] in 100 ns. Phase data for each bunch are recorded in a deep memory for diagnostics while the average phase of all the bunches in one turn is reported to the DSP. The magnitude data are used in an automatic gain control circuit to maximize the dynamic range of the $\mathrm{ADCs}$. The feedback equation is;

$$
\begin{aligned}
\delta \omega_{\mathrm{rf}}= & \mathrm{k}_{\varphi}\left(\varphi_{\text {bunch }}-\varphi_{\mathrm{s}}\right) \\
& +\mathrm{k}_{\mathrm{R}}\left(\mathrm{R}_{\text {Beam }}-\mathrm{R}_{\text {steering }}\right) \\
& +\mathrm{k}_{\mathrm{I}} \int\left(\mathrm{R}_{\text {Beam }}-\mathrm{R}_{\text {steering }}\right) \mathrm{dt}
\end{aligned}
$$

Where: $k_{\varphi}=1600 \mathrm{~Hz} / \mathrm{radian}, \mathrm{k}_{\mathrm{R}}=330 \mathrm{~Hz} / \mathrm{mm}, \mathrm{k}_{\mathrm{T}}=6400$ $\mathrm{Hz} / \mathrm{mm} / \mathrm{s}$. For the proton beam, which does not cross transition, the rings were locked in phase throughout the ramp by an additional term proportional the phase difference between the rings (measured at the revolution frequency). This prevents modulation of the beam-beam interaction during the ramp. The loops are open during the filling process and closed just before the ramp. When the loops close the averaged measured values of phase and radius are taken as the reference to the loops. Thereafter the reference values become the synchronous phase angle $\varphi_{s}$, and the radial steering command function $R_{\text {stering }}$ which updates at $720 \mathrm{~Hz}$. The loops open at the end of the ramp.

\section{Landau Cavities}

High intensity bunches $\left(-10^{11}\right.$ charges) in RHIC are clearly unstable, exhibiting shape oscillations without damping for many millions of turns.[7] Proton bunches are stabilized by switching on one of the $197 \mathrm{MHz}$ storage cavities at $100 \mathrm{kV}$ to enhance synchrotron frequency spread and provide Landau damping. This Landau cavity is used throughout the ramp.

\section{REBUCKETING}

In local jargon "rebucketing" [8] refers to the process of compressing the bunch in length and transferring it from the $28 \mathrm{MHz}$ system to the $197 \mathrm{MHz}$ storage system. A compression ratio of two or more is required. The technique of jumping to the unstable fixed point and back is most often used. The phase loop is active during the rebucketing to damp dipole oscillations (common cavities off). The precise set points for the relative phases of the storage cavities were obtained empirically by scanning the phases and measuring the synchrotron frequency to very high precision $(0.1 \%)$ from longitudinal Schottky signals at $2.7 \mathrm{GHz} .[9]$

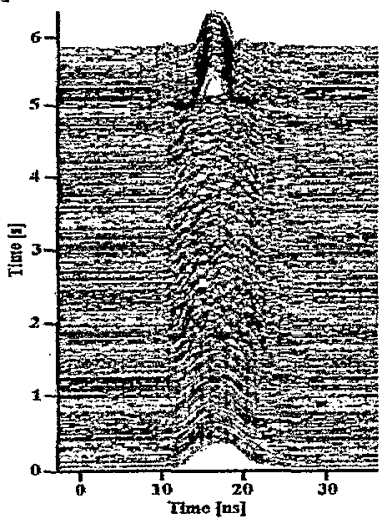

Figure 1. Evolution of the bunch shape during rebucheting. The bunch shape distortions begin 4 seconds before the gymnastic when the dampers are withdrawn.

\section{Strategy to combat beam load/emittance growth}

Beam loading is a major problem causing longitudinal emittance growth in the seconds just before rebucketing. When the storage cavities are at very low voltage while the tuning servo settles, a rapid onset of bunch shape distortions is seen (figure 1). The onset coincides with the withdrawal of the fundamental mode damper. Even though the cavity is equipped with high-gain of feedback the beam induced voltage overwhelms the generator induced voltage and the system is unstable. The beam loading problem was overcome by shaping the Fourier spectrum of the bunch current such that second node in the envelope of the spectrum occurs near $197 \mathrm{MHz}$. (figure 2) Since this is the state where the bunch is stretched the compression gymnastic becomes just to snap up the $28 \mathrm{MHz}$ voltage, and after $1 / 4$ synchrotron period switch on the $197 \mathrm{MHz}$ voltage to approximately $3 \mathrm{MV}$. Operationally this switch-on of the storage cavities $(10 \mathrm{kV}$ to $\sim 1 \mathrm{MV}$ in $1 \mathrm{~ms}$ ) proved to be the most difficult. To address this a new technique of "frequency-shift" rebucketing was tested. In this technique the storage cavities are turned on slowly at an offset frequency of 2 
$\mathrm{kHz}$ where they are de-coupled from the beam. At the rebucketing instant the frequency is jumped to the beam frequency. This is a minor transient for the cavities. because it is only a fraction of their natural bandwidth.

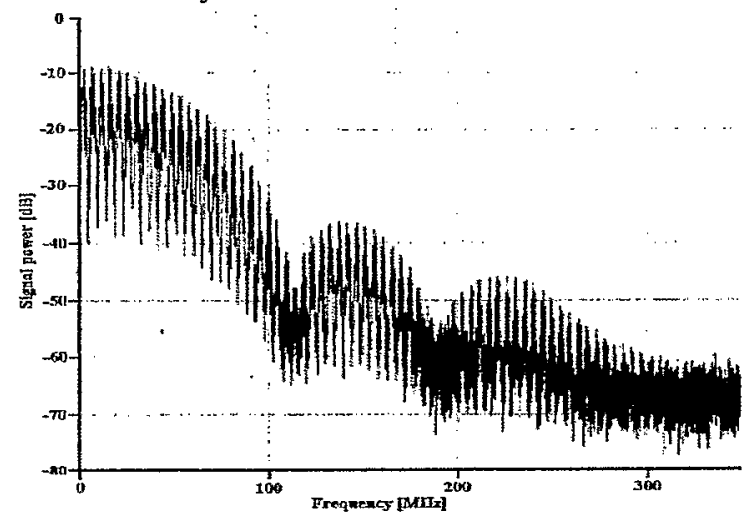

Figure 2. Spectrum of the deuteron beam current. The bunch has been stretched so that the second node is near the cavity frequency:

\section{Hardware improvements}

Luminosity lifetime improves with higher voltage in the storage cavities. The cavities of the storage system received a number of improvements before the latest run to allow for higher useable voltage. These included: water-cooling the ceramic windows of the power input coupler, rebuilding the solid state $2 \mathrm{~kW}$ driver amplifiers, installation of signal limiters to prevent overdrive at switch-on/off, and development of a software application to automate the high voltage conditioning of the cavities.

\section{COGGING}

Since the two rings of RHIC accelerate with independent if systems they arrive at store with unequal frequencies. To bring the beam into collision, first the beam feedback loops are switched open without a transient. Then the frequency of each ring is ramped at $1 \mathrm{~Hz} /$ second $(28 \mathrm{MHz}$ system) to a set point for the collision frequency. This frequency is chosen to give the best-centered orbits in the arcs of both rings. The rings share a master $10 \mathrm{MHz}$ clock for the Direct Digital Synthesizers and frequency control words are made exactly equal, thus locking the phase relationship between the bunches. The phase angle between the abort gaps is then measured automatically and a frequency excursion of $10 \mathrm{~Hz}$ is applied to one ring for a calculated time to align the abort gaps to within one bucket. Finally the rf phase between the vector sums of the acceleration cavities of the two rings is precisely measured with a LockIn amplifier (Standford Research, SR844) and a second frequency excursion aligns the collision spot to the Interaction Points. A longitudinal pickup at IP4 is used to calibrate the equipment. Since the same pickup measures both beams there is no systematic uncertainty in the collision point adjustment. The beams can be un-cogged and re-cogged at will (common cavities off) for machine experiments, such as beam-beam tune effects.
BUNCHED BEAM LIFETIME / RF NOISE

Intra-Beam Scattering is the fundamental limitation but rf noise can reduce bunched beam lifetime also. We are able to separate these effects in RHIC by comparing gold and deuterons upon which IBS has a negligible effect. An additional diagnostic tool is the fine structure of the longitudinal Schottky signals at high frequency. In the gold/deuteron run these techniques indicated a problem in the if source.

\section{Spectral purity of rf source}

The Schottky spectrum from deuterons showed a sharp line at $120 \mathrm{~Hz}$, which is within the band of the synchrotron frequencies. In fact, there was evidence of depopulation of the distribution in the neighborhood of the line. The strength of this line far exceeded the Schottky power, suggesting a coherent behavior for all bunches and thereby pointing to a problem with the spectral purity of the if source. A high dynamic range measurement of the $r f$ drive signal indeed revealed a line $120 \mathrm{~Hz}$ from the carrier ( $197 \mathrm{MHz}$ ) at $-72 \mathrm{dBc}$ in a $10 \mathrm{~Hz}$ bandwidth. The generator of this signal was the $H P 8644 B$, phase locked to $28 \mathrm{MHz}$. A new source was built using the direct synthesis technique with better spectral purity ($90 \mathrm{dBc}$ ) and the bunched beam lifetime improved substantially. The master $10 \mathrm{MHz}$ clock sets the ultimate noise floor. This is a Stanford Research FS725 Rubidium disciplined source with single sideband noise level less than $-130 \mathrm{dBc} / \mathrm{Hz}$ at $10 \mathrm{~Hz}$ offset from the carrier. We find that in practice the noise floor is dominated by the power supply noise of the VME crates that house the synthesizers.

\section{Power supply ripple in driver amplifier}

Another possible source of $\mathrm{rf}$ noise is power supply ripple in the $\mathrm{rf}$ drive chain. At the highest rf voltage the $2 \mathrm{~kW}$ solid state driver amplifiers are close to their maximum output. We found that their de regulators exhaust their compliance voltage and $180 \mathrm{~Hz}$ line frequency ripple modulates the if output. This frequency is especially damaging to the beam since the band of synchrotron frequencies encompasses this value. The power supplies $(120 \mathrm{~A} @ 60 \mathrm{~V})$ were rebuilt with more compliance.

\section{REFERENCES}

[1] S. Ozaki, RHIC Commissioning, IEEE PAC2001, Chicago

[2] F. Pilat, RHIC Status and Plans, Proc. EPAC 2002, Paris, pp15-19

[3] JML Brennan, et al. RF Beam Conirol for RHIC, Proe. EPAC1998, Stackholm, pp. 1705-1707

[4] J. DeLong, et al. Synthesizer Controlled Beam Transfer from AGS to RHIC, IEEE PAC2001, Chicago, pp.1523-1525

[5] C. Schultheiss, IM. Brennan, A State-Variale Description of the RHIC RF Control Loops, Proc. EPAC2002, Paris, pp.2097-2099

[6] R. Andraka, "A Survey of CORDIC Algorithms for FPGA based computers", FPGA 98, Monterey (1998). CA USA

[7] M Blaskiewicz, et al, Longitudinal Solitions .... these preceedings

[8] W.Pirkl, CERN, Private communication

[9] $M$. Blaskiewicz, et al. Longitudinal Impedance Measurements in RHIC, Froc. EPAC2002, Paris, pp:1488-1590 